\title{
Medical students raising concerns about staff members
}

\author{
Authors: Abhishek Kakkar and Damien Lynch
}

\section{Introduction}

Raising concerns is a fundamental responsibility of professional medical practice ${ }^{1}$ and plays an important role in ensuring patient, trainee, staff and organisational safety. The Francis Report ${ }^{2}$ and Bawa-Garba case highlight the importance of trainees being empowered to raise concerns.

Medical undergraduates are doctors in training. Education and training around raising concerns is a vital part of their professional development. Medical clinical educators (MCE) also act as role models for their students. There are occasions when MCE act unprofessionally. Anecdotal evidence suggests that there are barriers to medical students raising concerns in such instances. Very little is published about these perceived barriers. Lastly, there is little published experience around supporting undergraduate clinical educators dealing with concerns raised about them.

\section{Aims}

> Obtain feedback from students and MCE about the current 'raising concerns' process at East Lancashire Hospitals NHS Trust (ELHT) Department of Undergraduate Education.

$>$ Identify features of a process to support students raising concerns.

$>$ Identify features of a process to ensure MCE support when a concern is raised about them.

> Use the findings to develop an effective 'raising concerns' system for students and MCE alike, which is supportive and enables self-reflection and learning.

\section{Methods}

An anonymous online survey comprising multiple choice and white space questions was developed and sent to clinical students attending ELHT from Lancaster (LMS) and University of Central Lancashire (UCLan) medical schools, and ELHT MCE.

Questions related to barriers facing medical students when raising concerns about MCE and what the important features of a specific process would be. The responses were used to develop a specific 'raising concerns - MCE' flowchart to support students and educators. This was then implemented as a pilot.

\section{Results and discussion}

$26 / 79$ MCE responded (34\% response rate). Five out of 26 were aware of incidents of concerns about staff members reported by medical students, of whom three were directly involved. Of the 66 medical students from UCLan and 84 from LMS, 49 responded (33\%). Thirteen out of 49 were aware of, and seven were directly involved with, such incidents.

The results confirmed the findings of previous work on barriers facing students, ${ }^{3}$ namely fear of repercussions, undermining hierarchy, unsure of the process involved, and the perception that nothing would be done about it. There was scope for improvement in all cases that had arisen previously. Both MCE and students (92\% and $96 \%$ respectively) agreed the need for a consistent and systematic process. The suggestions from both groups as to what features a proposed system should have were identical; namely it should be fair, timely, supportive and should incorporate feedback for both the student and the MCE.

\section{Conclusion}

Concerns raised about MCE must be dealt with promptly and sensitively in order for both parties to understand and reflect on the circumstances leading to that complaint. The process we developed was designed in response to feedback from students and MCE. It is timely, with an aim to deal with the issue within 2 weeks. To address student worries about how to raise a concern, issues with hierarchy and the possibility of repercussions, we created a clear, consistent and fair framework which supports both parties. To aid learning, understanding and self-reflection, the process incorporates detailed feedback for both parties. A further quality improvement project studying the usefulness of this new system is currently being developed.

\section{References}

1 General Medical Council. Achieving good medical practice: guidance for medical students. Manchester: GMC, 2016. www.gmc-uk.org/ Achieving_good_medical_practice_0816.pdf_66086678.pdf [Accessed 22 March 2019].

2 Francis R. Report of the Mid Staffordshire NHS Foundation Trust public inquiry. London: TSO, 2013. www.gov.uk/government/publications/ report-of-the-mid-staffordshire-nhs-foundation-trust-public-inquiry [Accessed 22 March 2019].

3 Johnson L, Malik N, Gafson I et al. Improving patient safety by enhancing raising concerns at medical school. BMC Med Educ 2018;18:171. 